\title{
Análise do comportamento de promoção da saúde e do bem-estar pessoal em universitários
}

\author{
Analysis of the behavior of health and personal well-being promotion \\ among university students
}

\author{
Jordânia Rocha Franco, ${ }^{1}$ Manoel Borges da Silva Junior, ${ }^{2}$ Jose Wicto Pereira Borges, ${ }^{3}$ \\ Erisonval Saraiva da Silva ${ }^{4}$
}

\begin{abstract}
RESUMO
O objetivo deste artigo é analisar o comportamento de promoção da saúde e o bem-estar pessoal em universitários. Estudo transversal, quantitativo, realizado com 311 universitários de uma Instituição de Ensino Superior (IES) pública. Foram aplicadas as escalas de comportamento de promoção da saúde e de bem-estar pessoal da Nursing Outcomes Classification. Realizada análise descritiva e bivariada com aplicação da correlação de Pearson, adotando-se nível de significância de 5\%. Os universitários em sua maioria eram do sexo feminino $(70,1 \%)$ com média de idade de 23,8 $\pm 7,1$ anos; $32,2 \%$ entraram na universidade pelo sistema de cotas e $54,0 \%$ afirmaram ter renda familiar de até um salário mínimo. Os universitários demonstraram mediano comportamento de promoção da saúde com 68,5 $\pm 10,9$ pontos e de bem-estar pessoal com $46,8 \pm 9,2$ pontos. A correlação mostrou que 0 escore de comportamento de promoção de saúde aumenta à medida que o escore de bem-estar pessoal também aumenta $(r=0,32, p<0,005)$. $O$ estudo mostrou a relação entre o comportamento de promoção da saúde e o bem-estar pessoal de estudantes universitários. O conhecimento dessa relação permite novas possibilidades de intervenção nessas duas facetas importantes para o cuidado do público universitário, primordialmente constituído por adultos jovens. Os resultados poderão ser utilizados para a formulação de ações que impactem em incentivar os indicadores de promoção da saúde e bem-estar pessoal investigados.
\end{abstract}

\footnotetext{
${ }^{1}$ Graduada em Enfermagem pela Universidade Federal do Piauí (UFPI). E-mail: jordania_franco@hotmail.com

${ }^{2}$ Graduado em Enfermagem pela Universidade Federal do Piauí (UFPI).

${ }^{3}$ Doutor em Cuidados Clínicos em Saúde pela Universidade Estadual do Ceará (UECE). Professor permanente do Programa de Pós-graduação em Saúde e Comunidade (Mestrado) da Universidade Federal do Piauí (UFPI). ${ }^{4}$ Mestre em Terapia Intensiva pela Sociedade Brasileira de Terapia Intensiva (SOBRATI). Docente do Curso de Enfermagem da Universidade Federal do Piauí no Campus Amílcar Ferreira Sobral (CAFS/UFPI).
} 
PALAVRAS-CHAVE: Comportamento de risco. Estilo de vida. Promoção da saúde.

\section{ABSTRACT}

The aim is to analyze the behavior of health and personal well-being promotion among university students. This is a cross-sectional, quantitative study performed with 311 university students from a Public Higher Education Institution. The behavior scales of health and personal well-being promotion of the Nursing Outcomes Classification were applied. A descriptive and bivariate analysis was performed using Pearson's correlation, adopting a significance level of $5 \%$. The majority of the students were female (70.1\%) with an average age of $23.8 \pm 7.1$ years old; $32.2 \%$ entered university through the quota system and $54.0 \%$ reported having a family income of up to 1 minimum wage. The students showed a median behavior related to health promotion with $68.5 \pm 10.9$ points and personal well-being with $46.8 \pm 9.2$ points. The correlation showed that the Health Promotion Behavior score increases as the Personal Well-Being score also increases $(r=0.32, p<0.005)$. The study presented the relationship between health promotion behavior and the personal well-being of university students. The knowledge of this relation allows new possibilities of intervention in these two facets that are important for the care of the university public community, mainly constituted by young adults. The results can be used to formulate actions that have an impact on the encouragement of the indicators of the investigated health and personal well-being promotion.

KEYWORDS: Risk-taking. Life style. Health promotion.

\section{INTRODUÇÃO}

A experiência acadêmica se configura por momentos de transformações, desafios e dificuldades que precisam ser superadas pelo estudante universitário. Tratase de um período bastante peculiar na vida do indivíduo, pois é geralmente sincronizado com as mudanças e as adaptações próprias da transição da adolescência para a vida adulta. ${ }^{1,2,3}$

O contexto do Ensino Superior permite interações sociais diversas e exige do universitário muitas habilidades interpessoais e acadêmicas, talvez ainda não utilizadas anteriormente. ${ }^{4}$ A universidade representa esse período de transição, no qual, para esses jovens, envolve a adaptação de comportamentos em um ambiente novo. ${ }^{2}$

Durante o período acadêmico muitos universitários acabam desenvolvendo comportamentos de risco e pouco saudáveis, que podem comprometer tanto a saúde quanto a qualidade de vida. Esse período de mudança pode transformar o estilo de vida dessas pessoas no incremento de riscos relacionados a hábitos alimentares, ao sono, às práticas de exercícios físicos, ao consumo de álcool e tabaco e até mesmo ao uso de 
outras drogas. ${ }^{5}$ Ademais, alunos de graduação experimentam estresse e ansiedade significativos, inibindo o aprendizado e aumentando o desgaste. ${ }^{6,7}$

Nesse contexto de elevados fatores de risco, a reduzida adoção de comportamentos de promoção da saúde dos universitários influencia diretamente o bem-estar pessoal. Neste estudo, entende-se comportamento de promoção da saúde como ações pessoais para aumentar ou manter o bem-estar; e o bem-estar pessoal é definido como o alcance da percepção positiva da própria condição de saúde. ${ }^{8} \mathrm{O}$ bemestar pessoal está intimamente relacionado à condição de saúde do indivíduo e essa responsabilidade vai além do setor de saúde, exigindo por parte da população a escolha por um estilo de vida mais saudável. ${ }^{9}$

Nesse sentido, a ideia de promoção da saúde vem sendo discutida e abordada como proposta e estratégia para a melhoria das condições de saúde da população, atuando como um processo de capacitação e empoderamento da comunidade para a melhoria da saúde e da qualidade de vida, incluindo pessoas em processo educacional. ${ }^{10}$ Atua ainda como uma estratégia fundamental para impulsionar transformações nesses adultos jovens universitários e resgatar através do empoderamento, mudanças no cuidado das relações humanas e nas práticas de saúde.

Dessa maneira, para alcançar um potencial elevado de saúde, é necessário o estímulo para a adoção de hábitos de vida saudáveis, considerando os fatores pessoais de origem interna ou externa dos indivíduos, tais como o comportamento em situações de saúde-doença, percepções acerca da situação vivenciada ou mesmo o autocontrole sobre suas vidas. ${ }^{10}$ Além dos fatores pessoais, como trabalho e família, os estudantes dos cursos de graduação apresentam altos níveis de estresse e ansiedade em programas competitivos de estudo. ${ }^{7}$

Os comportamentos e as percepções que deles provém, podem contribuir positiva ou negativamente na definição de um estilo de vida e influenciar na saúde e no bem-estar. Além do exposto, salienta-se a importância do conhecimento da experiência de comportamento e bem-estar de estudantes no Ensino Superior, pois isso pode influenciar futuras decisões de carreira e pode ter um impacto no desgaste da profissão. ${ }^{11}$ Nessa perspectiva, questiona-se o que caracteriza o comportamento de promoção da saúde e do bem-estar pessoal de estudantes universitários e como eles se inter-relacionam. 
Dessa maneira, este estudo teve como objetivo analisar o comportamento de promoção da saúde e do bem-estar pessoal de universitários de uma Instituição de Ensino Superior (IES) pública no estado do Piauí. Conhecendo os aspectos comportamentais praticados por esses indivíduos, intervenções podem ser realizadas no sentido de modificar hábitos poucos saudáveis e melhorar o bem-estar dessa população através de atividades de promoção da saúde.

\section{MATERIAIS E MÉTODOS}

Estudo transversal, analítico, de natureza quantitativa, realizado em uma Instituição de Ensino Superior (IES) pública sediada na cidade de Floriano (PI). Essa IES faz parte do projeto de Reestruturação e Expansão da Universidade Federal do Piauí (REUNI), e serve de referência para estudantes da região dispondo dos cursos de Enfermagem, Administração, Pedagogia, Ciências Biológicas e Educação no Campo. A população foi composta por 1.420 universitários dos cinco cursos de graduação.

O cálculo de amostra foi realizado considerando os seguintes parâmetros: erro de $5 \%$, índice de confiança de $95 \%$ e considerando o desconhecimento da prevalência dos fatores de risco para o comportamento de promoção da saúde da população investigada firmou-se em 50\%. A amostra foi 311 universitários estratificada por curso.

Os critérios de inclusão foram: ter 18 anos ou mais e estar com matrícula ativa na IES. Foram excluídos os alunos que tivessem algum impedimento para obtenção das medidas antropométricas e gestantes.

A coleta dos dados foi realizada entre outubro de 2016 e janeiro de 2017. Foram coletadas as seguintes variáveis: idade, sexo, curso, ensino técnico, outra graduação, cotista, renda, estado civil e medidas antropométricas de peso e altura para cálculo do Índice de Massa Corporal (IMC), de circunferência da cintura (CC) e circunferência do quadril (CQ), bem como o comportamento de promoção da saúde e de bem-estar pessoal.

Os dados relativos ao peso foram obtidos com o indivíduo descalço, utilizandose uma balança portátil digital com capacidade para $150 \mathrm{~kg}$ e uma precisão de 0,1 kg. ${ }^{12}$ A altura foi avaliada com o uso de fita métrica com precisão de $0,5 \mathrm{~cm}$, aderida a uma parede sem rodapé. As aferições foram tomadas com os alunos descalços, de costas, 
com os pés juntos e os braços estendidos ao longo do corpo, em posição ereta e olhando para frente. ${ }^{13} \mathrm{O} I \mathrm{IMC}$ foi definido com base na razão entre o peso do universitário e o quadrado de sua altura. ${ }^{13}$

A verificação da CC foi realizada com fita métrica inextensível e com a roupa afastada, localizando-se a fita no ponto médio entre a crista ilíaca anterior superior e a última costela, sendo os valores normais considerados em $88 \mathrm{~cm}$ e $102 \mathrm{~cm}$ para mulheres e homens, respectivamente. ${ }^{13}$ Quanto à $\mathrm{CQ}$, tomou-se a medida do quadril, no seu maior diâmetro, com a fita métrica passando sobre os trocânteres maiores. ${ }^{14}$

Em relação ao comportamento de promoção da saúde e do bem-estar pessoal foram mensurados a partir da aplicação das escalas da Nursing Outcomes Classification: Escala de Comportamento de Promoção da Saúde (ECPS) composta por 20 indicadores acompanhados por uma escala adjetival de cinco pontos que vai de 1 (nunca demonstrado) a 5 (consistentemente demonstrado). Nela o universitário escolhe a resposta mais adequada para seu estilo de vida. Os resultados das avaliações dos indicadores da ECPS foram somados, sendo 20 pontos o valor que indica pouco comportamento de promoção da saúde e 100 pontos uma alta proficiência no comportamento. $^{8}$ Já a Escala de Bem-Estar Pessoal (EBEP) é composta por 13 indicadores acompanhados por uma escala adjetival de cinco pontos que vai de 1 (nem um pouco satisfeito) a 5 (completamente satisfeito). Os resultados das avaliações dos indicadores da EBEP foram somados, sendo 13 pontos o valor que indica pouco bemestar pessoal e 65 pontos uma alto nível de bem-estar. ${ }^{8}$

A análise dos dados foi realizada no SPSS versão 20.0, no qual foi possível verificar estatísticas descritivas para avaliar o desempenho dos universitários nos indicadores dos instrumentos utilizados, das frequências e das porcentagens de variáveis dicotômicas. Foi verificada a normalidade das variáveis através do teste de Kolmogorov-Smirnov e realizado teste de correlação de Pearson entre as variáveis de interesse. Todas as análises adotando-se nível de significância de $0,5 \%(p<0,05)$.

A pesquisa foi aprovada pelo Comitê de Ética em Pesquisa da Universidade Federal do Piauí (UFPI), sob parecer no 1.665.314 e CAE no 56330816.0.0000.5214, de acordo com o preconizado pela Resolução no 466/2012. 


\section{RESULTADOS}

A amostra foi composta em sua grande maioria por mulheres $(70,1 \%)$, com média de idade de 23,8 anos. A maioria dos universitários não tem ensino técnico $(59,8 \%)$ ou outra graduação $(93,6 \%)$, e somente $32,2 \%$ são cotistas. No que se refere à renda familiar, pouco mais da metade recebe até um salário mínimo (54\%). Quanto ao estado civil, $79,7 \%$ são solteiros; e $65,3 \%$ se autoconsideram de cor da pele parda (Tabela 1).

Tabela 1 - Distribuição de frequências, porcentagens, médias e desvios padrão, segundo as características sociodemográficas dos universitários de Floriano (PI), 2017

\begin{tabular}{|c|c|c|c|c|c|}
\hline & VARIÁVEL & n (311) & $\%$ & $x$ & DP \\
\hline \multirow{3}{*}{ SEXO } & Masculino & 93 & 29,9 & & \\
\hline & Feminino & 218 & 70,1 & & \\
\hline & $18-25$ anos & 237 & 76,2 & 23,8 & 7,1 \\
\hline \multirow{5}{*}{ IDADE } & $26-30$ anos & 37 & 11,9 & & \\
\hline & $31-40$ anos & 24 & 7,7 & & \\
\hline & $41-55$ anos & 13 & 4,2 & & \\
\hline & Enfermagem & 47 & 15,1 & & \\
\hline & Pedagogia & 84 & 27,0 & & \\
\hline \multirow[t]{3}{*}{ CURSO } & LEDOC & 42 & 13,5 & & \\
\hline & Administração & 71 & 22,8 & & \\
\hline & Biologia & 67 & 21,5 & & \\
\hline \multirow{2}{*}{ ENS. TÉCNICO } & Sim & 125 & 40,2 & & \\
\hline & Não & 186 & 59,8 & & \\
\hline OUTRA & Sim & 20 & 6,4 & & \\
\hline GRADUAÇÃO & Não & 291 & 93,6 & & \\
\hline \multirow{2}{*}{ COTISTA } & Sim & 100 & 32,2 & & \\
\hline & Não & 211 & 67,8 & & \\
\hline \multirow{3}{*}{ RENDA FAMILIAR } & Até 1 salário mínimo & 168 & 54,0 & & \\
\hline & $>1$ e $<3$ salários mínimos & 122 & 39,2 & 1256,1 & 1028,7 \\
\hline & > 3 salários mínimos & 21 & 6,8 & & \\
\hline
\end{tabular}


(Conclusão)

\begin{tabular}{|c|c|c|c|c|c|}
\hline & VARIÁVEL & n (311) & $\%$ & $x$ & DP \\
\hline \multirow{4}{*}{ ESTADO CIVIL } & Casado(a)/união consensual & 54 & 17,4 & & \\
\hline & Solteiro (a) & 248 & 79,7 & & \\
\hline & Viúvo (a) & 3 & 1,0 & & \\
\hline & Divorciado(a) & 6 & 1,9 & & \\
\hline \multirow{5}{*}{ COR DA PELE } & Parda & 203 & 65,3 & & \\
\hline & Branca & 29 & 9,3 & & \\
\hline & Negra/Preta & 67 & 21,5 & & \\
\hline & Amarela & 11 & 3,5 & & \\
\hline & Indígena & 1 & 0,3 & & \\
\hline
\end{tabular}

Legenda: $\dot{\mathrm{X}}=$ média; $\mathrm{DP}=$ Desvio padrão

Fonte: elaborada pelo autor - 2018

Em relação ao comportamento de promoção da saúde, a Tabela 2 apresenta as estatísticas descritivas dos indicadores utilizados na sua avaliação. $O$ indicador ato de evitar uso recreativo de drogas recebeu a maior média atribuída $(4,62)$. Já o uso de técnicas eficientes de redução do estresse, obteve a menor média $(2,46)$ e a menor mediana (2), indicando que pelo menos um quinto da amostra "raramente demostrava" comportamentos de controle do estresse.

Tabela 2 - Resultado dos itens avaliados na Escala de Comportamento de Promoção da Saúde (ECPS), em Floriano (PI), 2017

\begin{tabular}{lcccc}
\hline \multicolumn{1}{c}{ Indicadores } & Md & $\dot{\mathbf{X}}$ & DP & KS \\
\hline Uso de comportamentos para evitar riscos & 4 & 3,85 & 1,16 & $>0,001$ \\
$\begin{array}{l}\text { Monitorização do comportamento pessoal com relação } \\
\text { a riscos }\end{array}$ & 3 & 3,21 & 1,11 & $>0,001$ \\
Equilíbrio entre atividade e repouso & 3 & 2,99 & 0,98 & $>0,001$ \\
Manutenção de sono adequado & 3 & 3,13 & 1,07 & $>0,001$ \\
Uso de técnicas eficientes de redução do estresse & 2 & 2,46 & 1,13 & $>0,001$ \\
Manutenção de relações sociais & 4 & 3,82 & 0,97 & $>0,001$ \\
Desempenho rotineiro de comportamentos saudáveis & 3 & 3,16 & 0,93 & $>0,001$ \\
Apoio a políticas públicas saudáveis & 3 & 2,95 & 1,06 & $>0,001$ \\
Uso de apoio social para promover a saúde & 3 & 2,79 & 1,09 & $>0,001$ \\
Obtenção de imunizações recomendadas & 3 & 3,23 & 1,12 & $>0,001$ \\
\hline
\end{tabular}


(Conclusão)

\begin{tabular}{lcccc}
\hline \multicolumn{1}{c}{ Indicadores } & Md & $\dot{\mathbf{X}}$ & DP & KS \\
\hline Obtenção das avaliações de saúde recomendadas & 3 & 3,17 & 1,02 & $>0,001$ \\
Atendimento a uma dieta saudável & 3 & 2,82 & 1,09 & $>0,001$ \\
Ingestão de oito copos de água diários & 4 & 3,93 & 1,15 & $>0,001$ \\
Uso de estratégias eficazes de controle do peso & 3 & 2,98 & 1,25 & $>0,001$ \\
Uso de rotina eficaz de exercício & 3 & 2,79 & 1,35 & $>0,001$ \\
Ato de evitar exposições a doenças infecciosas & 4 & 3,92 & 1,07 & $>0,001$ \\
Ato de evitar exposição ao fumo passivo & 5 & 4,19 & 1,12 & $>0,001$ \\
Ato de evitar mau uso do álcool & 5 & 3,99 & 1,25 & $>0,001$ \\
Ato de evitar uso do tabaco & 5 & 4,46 & 1,19 & $>0,001$ \\
Ato de evitar uso recreativo de drogas & 5 & 4,62 & 1,03 & $>0,001$ \\
\hline
\end{tabular}

Legenda: $\mathrm{Md}$ = mediana; $\dot{\mathrm{X}}$ = média; DP = Desvio padrão; KS = Teste de Kolmogorov Smirnov

Fonte: elaborada pelo autor - 2018

Os resultados mostram que menos da metade dos universitários responderam que frequentemente ou consistentemente realizam os comportamentos de promoção da saúde. Os indicadores relacionados a evitar fumo passivo, mau uso do álcool e do tabaco e uso recreativo de drogas foram os únicos que os universitários responderam ter consistentemente.

Alguns indicadores relacionados à monitorização do comportamento de riscos, à rotina de comportamentos saudáveis, ao apoio a políticas públicas saudáveis, ao uso de apoio social para promover a saúde, as imunizações e as avaliações do estado de saúde mostraram fragilidade no comportamento de promoção da saúde dos universitários. 0 indicador que obteve menor mediana foi uso de técnicas eficientes de redução do estresse, demonstrando que muitos universitários raramente apresentam comportamentos de controle do estresse.

Na próxima página, a Tabela 3 apresenta as estatísticas descritivas dos indicadores utilizados na avaliação do bem-estar pessoal. 
Jordânia Rocha Franco, Manoel Borges da Silva Junior, Jose Wicto Pereira Borges, Erisonval Saraiva da Silva

Tabela 3 - Resultado dos itens avaliados na Escala de Bem-Estar Pessoal (EBEP), em Floriano (PI), 2017

\begin{tabular}{lcccc}
\hline \multicolumn{1}{c}{ Indicadores } & Md & $\dot{\mathbf{X}}$ & DP & KS \\
\hline Desempenho das atividades da vida diária & 5 & 4,48 & 0,79 & $>0,001$ \\
Desempenho dos papéis usuais & 4 & 3,75 & 0,92 & $>0,001$ \\
Saúde psicológica & 4 & 3,55 & 1,14 & $>0,001$ \\
Relações sociais & 4 & 3,89 & 0,96 & $>0,001$ \\
Vida espiritual & 4 & 3,70 & 1,10 & $>0,001$ \\
Saúde física & 3 & 3,27 & 1,09 & $>0,001$ \\
Estado cognitivo & 4 & 3,46 & 1,04 & $>0,001$ \\
Capacidade para enfrentar o cotidiano & 4 & 3,62 & 0,97 & $>0,001$ \\
Capacidade para relaxar & 3 & 3,28 & 1,20 & $>0,001$ \\
Nível de felicidade & 4 & 3,73 & 1,07 & $>0,001$ \\
Capacidade de expressar as emoções & 4 & 3,57 & 1,17 & $>0,001$ \\
Capacidade de controlar as atividades & 3 & 3,29 & 1,12 & $>0,001$ \\
Oportunidade de escolhas de cuidados de saúde & 3 & 3,20 & 1,13 & $>0,001$ \\
\hline
\end{tabular}

Legenda: Md = mediana; $\dot{\mathrm{X}}$ = média; DP = Desvio padrão; KS =: Teste de Kolmogorov Smirnov

Fonte: elaborada pelo autor - 2018

A grande maioria dos indicadores tiveram média abaixo de 4, demonstrando que os universitários estavam muito satisfeitos com esses indicadores de bem-estar pessoal. No entanto, apenas o desempenho das atividades da vida diária foi avaliado com "completamente satisfeitos" pela amostra. Outros indicadores que podem ser avaliados como positivos do bem-estar pessoal foram os relacionados ao desempenho dos papéis usuais, às relações sociais, à vida espiritual, ao enfrentamento do cotidiano e à felicidade.

Os indicadores que apresentaram maior impacto na redução do bem-estar pessoal foram saúde psicológica e física, estado cognitivo e capacidade para relaxar, expressar as emoções, controlar atividades e escolhas de cuidados de saúde.

O resultado da ECPS apresentou média de 68,5 ( $\pm 10,9 D P$, min 39 e máx 95$)$ pontos; e a de bem-estar pessoal, média de 46,8 ( $\pm 9,2 \mathrm{DP}$, min 21 e máx 65$)$ pontos. 0 Gráfico 1 apresenta a correlação entre o comportamento de promoção da saúde e o bem-estar pessoal. 
Gráfico 1 - Correlação entre bem-estar pessoal e comportamento de promoção da saúde em Floriano (PI), 2017

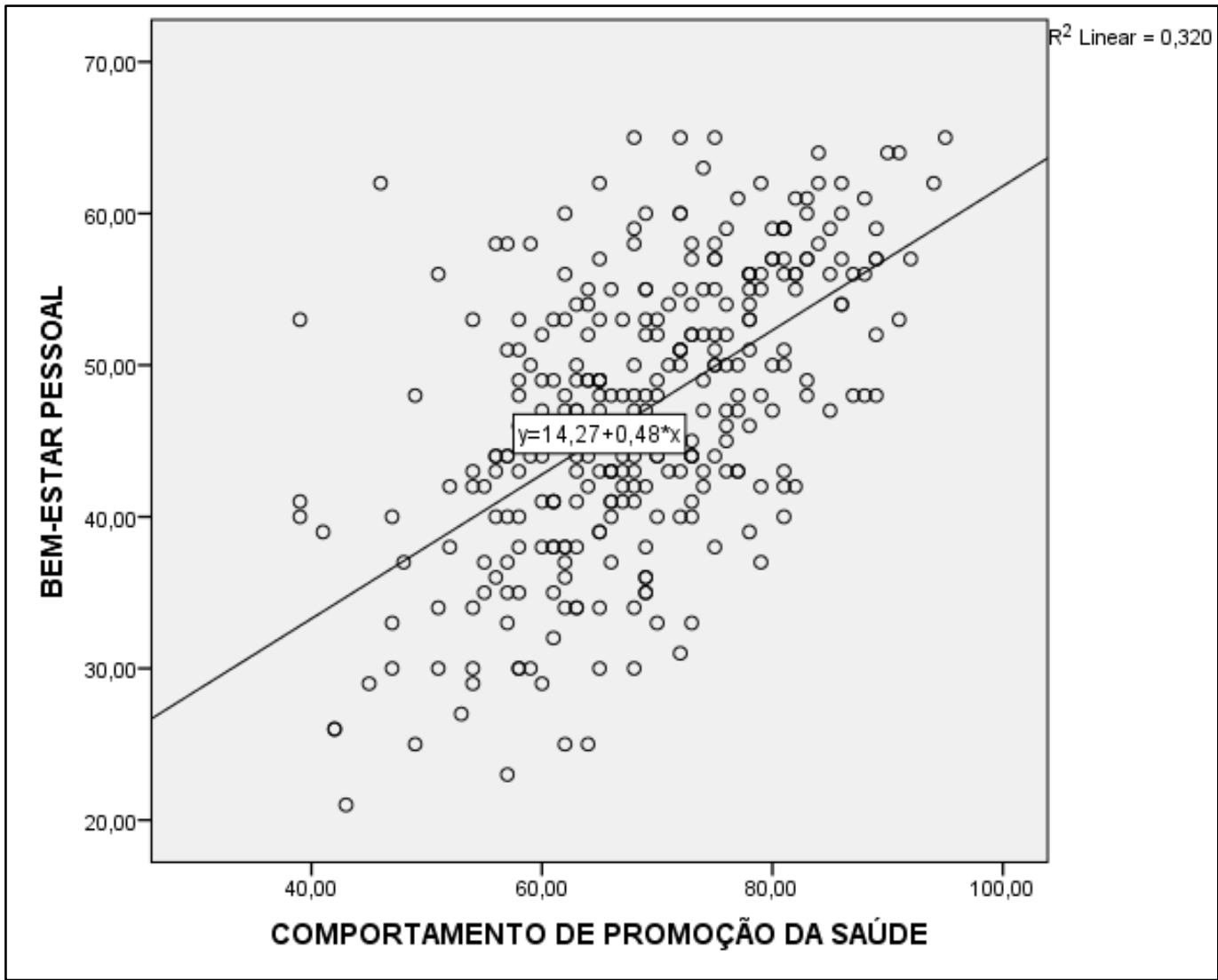

Fonte: elaborada pelo autor - 2018

O Gráfico 1 mostra o coeficiente de Pearson fraco positivo $r=0,32$ entre o bemestar e o comportamento de promoção da saúde. É possível afirmar que o escore de comportamento de promoção de saúde aumenta à medida que o escore de bem-estar pessoal também aumenta, revelando uma correlação positiva e significante entre essas variáveis.

\section{DISCUSSÃO}

Os resultados mostraram, através de alguns indicadores, baixo comportamento de promoção da saúde e insatisfação com o bem-estar pessoal nos universitários. Comportamentos de risco à saúde na vida universitária vêm sendo demonstrados em outros estudos, ${ }^{15,16,17}$ e podem estar relacionados ao perfil dessa parcela da população.

O perfil encontrado na maioria dos universitários foi sexo feminino, adultos 
jovens, solteiros, que não têm outra graduação e com renda familiar de até um salário mínimo. Nesse sentido, argumenta-se que o sexo feminino é o que mais tem participado de pesquisas no âmbito da saúde ${ }^{15} \mathrm{e}$ isso pode estar relacionado ao fato de as mulheres serem mais preocupadas com a saúde quando comparadas ao sexo masculino. ${ }^{19}$

Em relação à média de idade e o estado civil encontrado, o estudo constatou que, em sua grande maioria, estudantes de nível superior são formados por um público jovem e sem parceiros fixo. ${ }^{17}$

Estudos apontam que quanto mais afetos positivos, mais a pessoa estaria satisfeita com a vida. ${ }^{19}$ Outro estudo com jovens universitários, com média de idade de 21 anos, sugere que pessoas que vivenciam relacionamentos mais frequentes (namoro, casamento, entre outros), se mostram mais satisfeitas com a vida e também apresentam expectativas mais positivas em relação ao futuro, além de demonstrarem autoestima e bem-estar mais elevados. ${ }^{20}$

Em relação à renda, o relatório socioeconômico e cultural dos estudantes de graduação das universidades federais brasileiras mostrou que praticamente metade deles pertence às classes populares, o que contradiz a ideia de que tal instituição seja composta, em sua grande maioria, por um público de classe média alta. ${ }^{21}$

Sobre o comportamento de promoção da saúde e bem-estar pessoal, o estudo revelou médias elevadas em alguns escores das duas escalas. Esse achado faz surgir uma preocupação em relação a esses comportamentos, uma vez que uma parcela significativa da população de universitários não apresenta padrões considerados saudáveis. ${ }^{22}$

Foi realizado um estudo com universitários em Minas Gerais sobre comportamentos saudáveis evidenciando que 95,4\% apresentavam desempenhos de risco no que se refere aos hábitos alimentares, ao consumo de bebidas alcoólicas, à ausência/carência de exercícios físicos e ao gasto excessivo de tempo com eletrônicos. ${ }^{23}$

O universo acadêmico pode oferecer um ambiente propício à adesão e ao desenvolvimento de maus hábitos alimentares e um estilo de vida inadequado, podendo resultar em implicações negativas, formando um grupo vulnerável a riscos significativos com relação à saúde.

Em relação ao bem-estar pessoal dos universitários, estudos perceberam que experiências universitárias, incluindo as vivências pessoais, interpessoais, de carreira, 
estudo e institucionais mais satisfatórias podem produzir uma maior sensação de bemestar pessoal. ${ }^{24}$

Esse ponto de vista vai ao encontro de outro estudo que encontrou resultados semelhantes, nos quais uma boa percepção de saúde e hábitos de vida saudáveis são excelentes ferramentas para uma melhora positiva da qualidade de vida dos universitários, principalmente no domínio físico e nas relações sociais. ${ }^{25}$

Conjectura-se que o bem-estar pessoal se apresenta no mesmo sentido da qualidade de vida, assim, níveis insatisfatórios de qualidade de vida podem impactar negativamente a saúde mental dos estudantes, bem como gerar dificuldades no processo de ensino-aprendizagem. ${ }^{26}$ Estudo realizado com estudantes de graduação em Enfermagem na China encontrou altos níveis de estresse e morbidade psicológica no último do ano do curso. ${ }^{11} \mathrm{Um}$ estudo de revisão envolvendo 26 estudos de todo o mundo mostrou a influência da ansiedade e do estresse no desempenho acadêmico de alunos de Enfermagem. ${ }^{7}$

Como apresentado nos resultados, quanto maior o grau de vulnerabilidade a comportamentos de risco em universitários, maior a insatisfação em relação ao bemestar pessoal. Apesar de se tratar de um público com conhecimento básico acerca dos riscos que esses comportamentos podem acarretar para a saúde, contudo, trata-se ainda de uma realidade bem característica entre o público jovem.

Em relação aos resultados dos indicadores da ECPS, podemos perceber um resultado positivo, uma vez que grande parte dos universitários tem comportamentos saudáveis. Os únicos indicadores que apresentaram resultados negativos foram em relação ao uso de drogas lícitas e ilícitas: essa implicação é similar ao de alguns estudos encontrados na literatura que nos mostram que a universidade se configura como um ambiente propício à adesão desses comportamentos de risco. ${ }^{27,28}$

Um estudo realizado com universitários constatou que 19\% deles, em diferentes regiões do país, fazem consumo frequente de bebidas alcoólicas, 48,7\% já experimentaram drogas ilícitas e $26 \%$ informaram uso de mais de uma droga no último ano. $^{27}$

Grande parte do consumo de drogas e suas implicações de gênero, sociais e legais entre universitários ocorrem em decorrência dos estresses das tarefas escolares 
e também para obter relaxamento físico e mental e/ou como medida alternativa de obtenção de bem-estar pessoal, inclusive dentro da própria universidade. ${ }^{28}$

Por outro lado, o indicador com menor desempenho entre os universitários que versa sobre a redução do estresse, é similar ao resultado encontrado em um estudo com universitários feito na Arábia Saudita, que evidenciou uma alta prevalência de estresse entre acadêmicos do curso de Medicina (90\%); e as medidas de enfrentamento mais empregadas por eles para o controle era de evitação ou fuga, ratificando que parte dos estudantes não procura meios alternativos de reduzi-los. ${ }^{29}$ Esses dados nos revelam a necessidade de os universitários contarem com uma maior atenção psicológica durante o decorrer da graduação.

Acerca da saúde psicológica, o mesmo estudo também mostrou que $36,4 \%$ dos universitários disseram já terem suas vidas acadêmicas prejudicadas por questões emocionais e $38,5 \%$ afirmaram ter sentido alguma dificuldade ou crise emocional durante o curso que dificultou no desempenho acadêmico. ${ }^{30}$

Assim como o estudo citado, o relatório das universidades federais identificou que $43 \%$ dos universitários que participaram desse levantamento apresentaram dificuldades significativas de adaptações às novas situações do universo acadêmico. ${ }^{21}$

Em relação ao bem-estar pessoal, as oportunidades de escolhas de cuidados de saúde são essenciais para a melhoria das condições de saúde. Os sistemas que asseguram o acesso a bens básicos promovem uma melhoria significativa no bem-estar físico e psicológico. ${ }^{31}$

Quando as oportunidades de escolhas de cuidados em saúde são acessíveis e resolutivas para os universitários que se configuram como um grupo vulnerável, melhor a percepção deles acerca do bem-estar físico e também psicológico, uma vez que sua percepção de saúde é ampliada.

Assim, evidencia-se a necessidade de desenvolvimento de ações especiais diante das diferentes demandas estudantis, a exemplo do monitoramento de indicadores, além de ações que auxiliem na mudança do comportamento e, possivelmente, melhorem a satisfação do bem-estar pessoal. 


\section{CONSIDERAÇÕES FINAIS}

O estudo mostrou a relação entre o comportamento de promoção da saúde e o bem-estar pessoal de estudantes universitários. O conhecimento dessa relação permite novas possibilidades de intervenção em duas facetas importantes para o cuidado do público universitário, primordialmente constituídos por adultos jovens. Os resultados poderão ser utilizados para a formulação de ações que impactem em incentivar os indicadores de promoção da saúde e bem-estar pessoal investigados. Promover comportamentos de saúde permitirá a adoção de práticas saudáveis de vida, acarretando na diminuição do risco de doenças crônicas não transmissíveis ao mesmo tempo que permitirá acessar a esfera psicoemocional do bem-estar desses indivíduos.

Entre as limitações encontradas nesse estudo, está o fato de o desfecho ter sido avaliado por meio de respostas obtidas através de um questionário autoaplicado, pois essa técnica pode sofrer viés de memória dos respondentes. Tal fato foi minimizado com as orientações prévias ao momento de preenchimento dos instrumentos.

Sugere-se o investimento em mais pesquisas sobre o tema e a implantação de ações mais efetivas através de ações educativas, contribuindo assim para a promoção da saúde, bem como para gerar uma melhor satisfação de bem-estar pessoal nessa população.

\section{REFERÊNCIAS}

1. Mercuri E, Polydoro SAJ. Estudante universitário: características e experiências de formação. Cabral Editora e Livraria Universitária, 2004.

2. Lira Neto JCG, Silva AP, Costa EPN, Lacerda MGC, Silva ARV, Freitas RWJF. Analysis of overweight and obesity in university students. Rev Enferm UFPE on line. 2012; 6 (11): 2770-6.

3. Santiago JCS, Moreira TMM, Florencio RS. Associação entre excesso de peso e características de adultos jovens escolares: subsídios ao cuidado de enfermagem. Rev. Latino-Am. Enfermagem. 2015; 23 (2): 250-8.

4. Bolsoni-Silva AT, Guerra BT. O impacto da depressão para as interações sociais de universitários. Estud. Pesqui. Psicol. 2014; 14 (2): 429-452.

5. Silva EC, Heleno MGV. Qualidade de vida e bem-estar subjetivo de estudantes universitários. Revista Psicologia e Saúde. 2012; 4 (1). 
6. Yıldırım N, Karaca A, Cangur S, Acıkgoz F, Akkus D. The relationship between educational stress, stress coping, self-esteem, social support, and health status among nursing students in Turkey: a structural equation modeling approach. Nurse Education Today. 2016; (48): 33-39.

7. Turner K, McCarthy V.L. Stress and anxiety among nursing students: a review of intervention strategies in literature between 2009 and 2015. Nurse Education in Practice. 2016; (22): 21-29.

8. Moorhead S, Johnson M, Maas ML, Swanson E. Nursing Outcomes Classification (NOC): measurement of health outcomes. 4th ed. Philadelphia: Elsevier; 2010.

9. Backes MTS, Rosa LM, Fernandes GCM, Becker SG, Meirelles BHS, Santos SMA. Conceitos de saúde e doença ao longo da história sob o olhar epidemiológico e antropológico. Rev. Enferm. UERJ. 2009 jan/mar; 17 (1): 111-7.

10. Sampaio FAA, Melo RP, Rolim ILTP, Siqueira RC, Ximenes LB, Lopes MVO. Avaliação do comportamento de promoção da saúde em portadores de diabetes mellitus. Acta Paul Enferm, 2008; 21 (1): 84-8.

11. Yang F, Smith GD. Stress, resilience and psychological well-being in Chinese undergraduate nursing students. Nurse Education Today. 2016; 49: 90-95.

12. Sulinga $E$, Koziel D, Gluszek S. Prevalence of metabolic syndrome in normal weight individuals. Ann Agric Environ Med. 2016; 23 (4): 631-5.

13. Freitas PP, Assunção AA, Bassi IB, Lopes AC. Overweight and workplace in municipal public setor. Rev Nutr. 2016; 29 (4): 519-27.

14. Associação Brasileira para o Estudo da Obesidade e da Síndrome Metabólica. Diretrizes brasileiras de obesidade (ABESO). São Paulo, SP - 2016, 4ํed.

15. Brito BJQ, Gordia AP, Quadros TMB. Estilo de vida de estudantes universitários: estudo de acompanhamento durante os dois primeiros anos do curso de graduação. Medicina (Ribeirão Preto). 2016; 49 (4): 293-302.

16. Vargas LM, Redkva PE, Cantorani JRH, Gutierrez GL. Estilo de vida e fatores associados em estudantes universitários de educação física. Revista de Atenção à Saúde. 2015 abr./jun; 13 (44): 17-26.

17. Sousa TF, José HPM, Barbosa AR. Condutas negativas à saúde em estudantes universitários brasileiros. Ciência \& Saúde Coletiva. 2013; 18 (12): 3563-3575.

18. Santos AKGV, Reis CC, Chaud DMA, Morimoto JM. Qualidade de vida e alimentação de estudantes universitários que moram na região central de São Paulo sem a presença dos pais ou responsáveis. Rev. Simbio-Logias. 2014; 7 (10).

19. Scorsolini-Comin F, Santos MA. Correlations between subjective well-being, dyadic adjustment and marital satisfaction in Brazilian married people. The Spanish Journal of Psychology, 2012; 15 (1): 166-176.

20. Zanon C, Bastianell MR, Pacico JC, Hutz CS. Desenvolvimento e validação de uma escala de afetos positivos e negativos. Psico-USF. 2013 mai/ago; 18 (2) 193-202.

21. Fórum Nacional de Pró-reitores de Assuntos Comunitários e Estudantis (FONAPRACE). Revista Comemorativa 25 Anos: histórias, memórias e múltiplos olhares. 2012. 
22. Oliveira NRC, Padovani RC. Saúde do estudante universitário: uma questão para reflexão. Artas Letters, 2014; 19 (3).

23. Crepaldi BVC, Guimarães HPN, Barbosa CD, Molina LS, Nogueira LMM, Soares LP. Elevada prevalência de fatores de risco para doenças crônicas entre universitários. Ciência \& Saúde. 2016; 9 (3): 135-143.

24. Rozin AB, Zanon C, Teixeira MAP. Bem-estar subjetivo, personalidade e vivências acadêmicas em estudantes universitários. Interação Psicol. 2014 jan./abr; 18 (1): 1-12.

25. Carleto CT. Hábitos de saúde e qualidade de vida de universitários da área da saúde [Dissertação]. Uberaba: Universidade Federal do Triângulo Mineiro: 2012.

26. Catunda MAP, Ruiz VM. Qualidade de vida de universitários. Pensamento Plural: Revista Científica. 2008; 2 (1).

27. Azevedo RCS. Uso de drogas por universitários. Revista Ensino Superior - UNICAMP. 2013; (11).

28. Dázio EMR, Zago MMF, Fava SMCL. Uso de álcool e outras drogas entre universitários do sexo masculino e seus significados. Rev Esc Enferm USP, 2016; 50 (5): 786-792.

29. Bassols AMS, Carneiro BB, Guimarães GC, Okabayashi LMS, Carvalho LG, Silva AB et al. Stress and coping in a sample of medical students in Brazil. Arch Clin Psychiatry. 2015; 42 (1): 1-5.

30. Silva EC. Qualidade de vida e bem-estar subjetivo de estudantes universitários. [Dissertação]. São Bernardo do Campo. Universidade Metodista de São Paulo, 2012.

31. Carvalho Al. Determinantes sociais, econômicos e ambientais da saúde. Fundação Oswaldo Cruz. 2013; 2, 19-38.

Submissão: maio de 2018.

Aprovação: setembro de 2018. 